\title{
Independent Motion Detection Directly from Compressed Surveillance Video
}

\author{
Zhongfei (Mark) Zhang \\ Computer Science Department \\ SUNY Binghamton \\ Binghamton, NY 13902, USA \\ zzhang@binghamton.edu
}

\author{
Stoyan Kurtev \\ International University - Bremen \\ Campus Ring 1 \\ 28759 Bremen, Germany \\ s.kurtev@iu-bremen.de
}

\begin{abstract}
This paper presents a novel solution to the problem frequently encountered in military/intelligence surveillance: automatic mining video data for independently moving targets. Instead of attempting to detect each individual independently moving target in each frame, this method focuses on determining whether or not each frame has independent motion. Consequently, it differs from the existing literature on this topic in the following two aspects: (1) fast detection (2) detection directly from the compressed video streams instead of from the image sequences. These two aspects are motivated from the applications that this research aims to address, and therefore, are the contributions to the literature of independent motion detection. The solution is based on the Linear System Consistency Analysis, and thus is called LSCA. A number of distinctive technical advantages of LSCA as compared with the existing methods in the literature are identified. Evaluations from both simulated and real data show that LSCA holds a great promise in achieving fast detection without sacrificing the reasonable detection accuracy.
\end{abstract}

\section{Categories and Subject Descriptors}

I.2.10 [Vision and Scene Understanding]: Motion; I.4.8 [Scene Analysis]: Motion; I.5.4 [Applications]: Computer Vision; H.3.3 [Information Search and Retrieval]: Information Filtering, Retrieval Models; H.5.1 [Multimedia Information Systems]: Video; I.1.2 [Algorithms]: Algebraic Algorithms

\section{General Terms}

Algorithms, Design, Measurement

\section{Keywords}

Independent Motion Detection, Surveillance Video, Linear

Permission to make digital or hard copies of all or part of this work for personal or classroom use is granted without fee provided that copies are not made or distributed for profit or commercial advantage and that copies bear this notice and the full citation on the first page. To copy otherwise, to republish, to post on servers or to redistribute to lists, requires prior specific permission and/or a fee.

IWVS'03, November 7, 2003, Berkeley, California, USA.

Copyright 2003 ACM 1-58113-780-X/03/00011 ...\$5.00.
System Consistency Analysis (LSCA), Singular Value Decomposition (SVD), MPEG

\section{INTRODUCTION}

This paper presents an approach to the problem of automatic detection of independently moving targets in a potentially large collection of surveillance video data obtained from an unmanned sensor (such as a video camear in an unmanned aerial vehicle). Consequently, this work is about Independent Motion Detection. This is not yet another paper on this topic. We take the problem from a new perspective and focus on two aspects of this problem: (1) fast detection (2) detection directly from the compressed MPEG video streams instead of from the image sequences. These two aspects are motivated from the applications that this research project aims to address. Therefore, the contributions of this work are reflected in these two aspects.

An object in motion from a surveillance video may be interpreted as a potential suspicious activity. In law enforcement applications, if the surveillance video is from a camera monitoring a highly access-restricted area (e.g., a cash reservoir in a bank), noticeable moving targets in the video may be related to possible activities such as robbery, or terrorist attacks. In military/intelligence applications, if the surveillance video is obtained from an unmanned aerial vehicle monitoring an enemy's military area, noticeable moving targets in the video may be related to activities such as military maneuvers. In both cases, if the camera is still, the problem of automatic detection of motion from the video is relatively easy to tackle with. However, in many applications, it is not possible to have a still camera. An example is in the automatic data collection in military surveillance using unmanned aerial vehicles, such as US Predators. In this case, the goal of the surveillance is to detect any military maneuvers, which are typically manifested as the target motion in the video. Note that since in this case the cameras are also in motion, the problem of detecting any target motion is consequently translated to the problem of detecting independent motion - the motion other than the camera motion. Fig. 1 shows exemplary frames from the real US Predator surveillance videos that represent two different scenarios: a scene with independent motion and a scene without independent motion. Due to the fact of large scale, automatic data collection (multiple Predators in nonstop data collection) in a typical military surveillance, the data volume is massive. Manual detection of independent motion is painfully proven to be extremely tedious and very 
expensive. Consequently, a solution to automatically mining the massive video data directly in a compressed storage format such as MPEG to retrieve the shots with independent motion is highly desirable. Motivated by this demand, an efficient and effective video data scanning algorithm is developed in this research for independent motion detection directly from compressed surveillance video.

\section{BACKGROUND AND RELATED WORK}

When a surveillance video is taken from a camera that is also in motion, every pixel in a frame may contain motion. For those background pixels, the motion reflected in the image domain corresponds to the $3 \mathrm{D}$ camera motion. On the other hand, for those pixels corresponding to independently moving objects in the frame, their motion corresponds to the combination of the $3 \mathrm{D}$ camera motion and their own independent motion in the 3D space. In this case, simple frame based differencing does not work [19], and certain sophisticated techniques must be applied to separate the independent motion from the camera motion, which is also called the background motion. This problem becomes even more complicated when there is $3 \mathrm{D}$ motion parallax involved. In this case, a $3 \mathrm{D}$ motion model must be applied in order to robustly and accurately separate the independent motion from the camera motion. Therefore, the problem of detection of independently moving objects here is reduced to the problem of independent motion detection.

There are two scenarios related to independent motion detection. Given a video stream or an image sequence, one scenario refers to the detection in which a temporal segmentation is conducted into those subsequences (called shots) that contain the scene in which one or more independently moving targets are present, in addition to a spatial segmentation and delineation of each of the independently moving targets in each of the frames of these shots. The other scenario, on the other hand, refers to the detection in which only the temporal segmentation is conducted to return those shots that contain independent motion; no spatial segmentation is performed to identify the independently moving targets in each frame. The focus of this paper is primarily in the latter approach. Taking the example of the US military surveillance data shown in Fig. 1, once the shots containing independent motion are automatically detected and retrieved, the major painstaking and tedious mining effort (i.e., manual searching the massive video data to detect those shots containing independent motion) is saved because the majority of the video does not have independent motion. Therefore, in terms of detecting independent motion in these applications, the objective is for temporal detection of the video shots containing independent motion, as opposed to spatial segmentation and delineation of each target in all the frames.

Motion analysis has been a focused topic in computer vision and image understanding research for many years [42, $18,15,12,27]$. Due to the difficult nature of the problems in this topic, it is still considered as an open area and many research efforts are still being paid in this topic $[10,11]$. Independent motion analysis, on the other hand, deals with multiple motion components simultaneously, and therefore, is presumably more challenging.

The earliest work in independent motion detection may be dated back to the early 80 's. Jain [21] proposed a solution assuming that the camera was under a translation. Adiv [1] assumed the availability of optical flow and used the flow to group regions based on the rigidity constraint over two frames. Nelson [29] proposed two methods based on velocity constraints to detect independently moving objects. Thompson et al [40] used a similar approach based on the rigidity constraint. Bouthemy and Francois [9] treated the problem of independent motion detection as a statistical regularization problem and attempted to use Markov Random Field model to solve for the problem. Ayer et al [7] used robust statistical regression techniques to detect independent motion. Smith and Brady [38] used geometric constraints for independent motion segmentation. Sharma and Aloimonos [35] provided a solution to this problem based on normal flow field - the spatiotemporal derivatives of the image intensity function, as opposed to the typical optical flow field. Irani and Anandan [19] proposed a three-frames constraint based on a general 3D motion parallax model to detect independent motion. Recently, Argyros et al [5, 3, 4, 6] and Lourakis et al [25] used stereo camera streams to detect independent motion. Their techniques were essentially the combination of applying the normal flow field to the stereo streams and using robust statistical regression. Fejes and Davis [13] developed a low-dimensional, projection-based algorithm to separate independent motion using the epipolar structure of rigid 3D motion flow fields. Torr [41] proposed a method based on model selection and segmentation for separating multiple 3D motion components. Pless et al [30] provided a solution to the problem in a special case in which the scene may be approximated as a plane, which is valid for typical aerial surveillance. Their method is based on spatiotemporal intensity gradient measurements to directly compute an exact background motion model, and then the independent motion is detected based on the constraint violation for the mosaics developed over many frames. Sawhney et al [34] proposed a method that simultaneously exploits both constraints of epipolar and shape constancy over multiple frames. This method is based on the previous work on plane-plus-parallax decomposition [22, 33, 36], and thus requires explicitly estimating the epipolar and the homography between a pair of frames.

Most of the existing techniques for independent motion detection in the literature require spatial segmentation (i.e., identification) of the independently moving targets in the frames or images. Due to this fact, very few of them can afford fast detection (such as real time or even faster than real time detection), as their solutions to temporal independent motion detection depend on the spatial independent motion segmentations. While these approaches are useful in general, due to the specific applications that have motivated this project, in order to deliver a fast detection, we only concern with detecting those video shots that contain independent motion, without specifically identifying the independently moving targets in the frames. We argue that it is not necessary to identify the moving targets in the image frames in the applications that we concern with. This is based on the following two reasons. (i) In the military, intelligence, as well as law enforcement applications, the time issue, i.e., the detection speed, is always an important concern. Obviously the spatial domain identification requires more processing time. (ii) It is not necessary to take the spatial domain identification approaches in these applications. Even if the independently moving targets are all segmented and identified in each frame, given the current status 

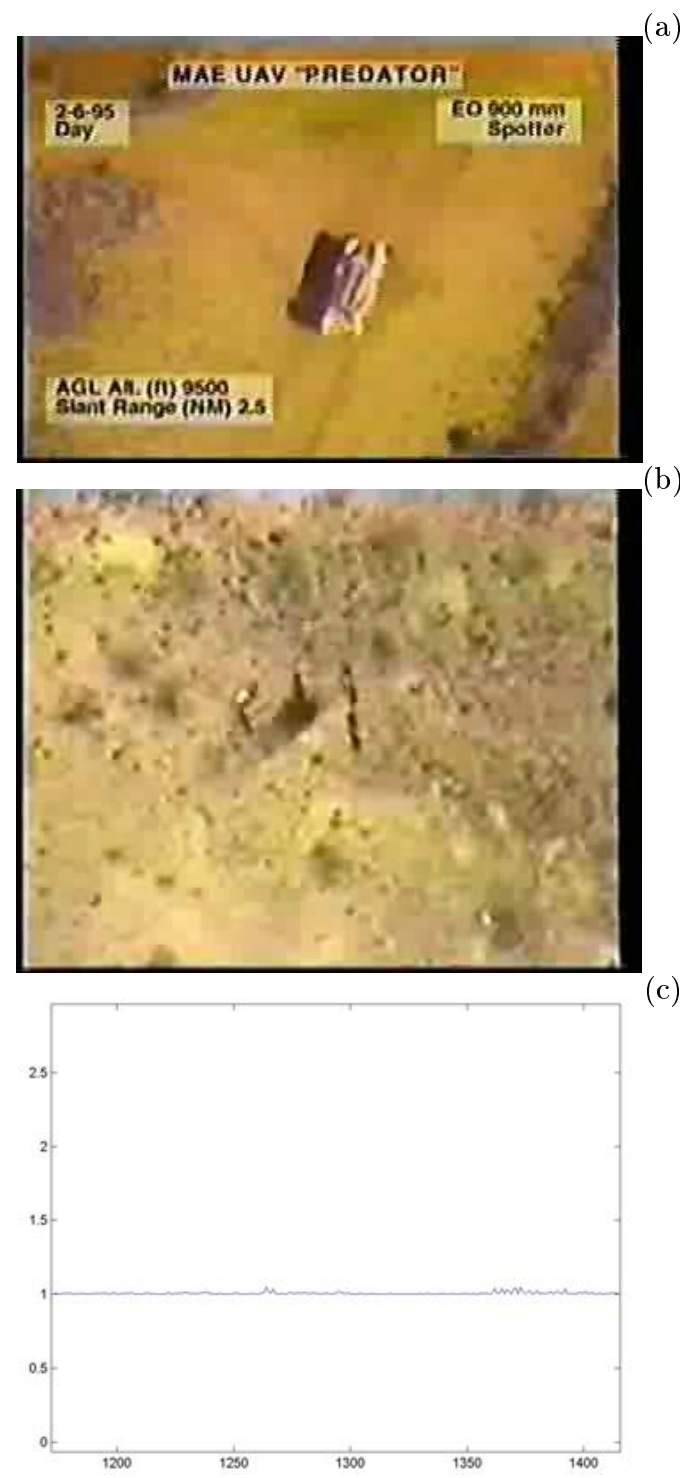

(c)

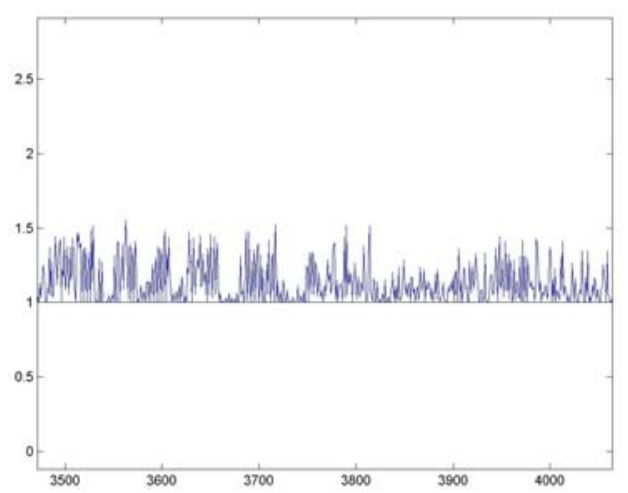

(d)

Figure 1: (a) An example of a shot containing an independently moving object (a missile launcher) (b) An example of a shot containing no independent motion (terrain) (c) The original $R$ statistics computed for the shot in (a) (1119 frames) (d) The original $R$ statistics computed for the shot in (b) (1058 frames). 
of computer vision and artificial intelligence in general, it is not possible to have a fully automated capability to interpret whether the segmented and identified independent motion in the frames indicates any military or intelligence significance without human expertise' interaction. Therefore, these detected shots must be sent to the Image Analysts for further analysis anyway, regardless of whether or not the independently moving targets are segmented and identified in each frames in these shots.

The other observation is that in the literature, most of the existing techniques for independent motion detection are based on image sequences, as opposed to compressed video streams. In other words, given a video, such as a surveillance video, these methods require that the video must be first fully decompressed to recover an image sequence before these methods can be applied. This restriction (or assumption) significantly hinders these techniques from practical applications, as in today's world, information volume grows explosively, and all the video sequences are archived in compressed forms. This is particularly true in the surveillance applications this paper is concerned with, in which the data volume is massive and they must be archived in a compressed form, such as MPEG.

Based on these considerations, we have developed a new approach to solving for the problem of fast independent motion detection directly from the compressed surveillance video streams. By fast, it is meant that the data scanning speed is the same or even faster than the real-time performance. This capability allows two possible application scenarios for this technology. The first is to equip the sensors with this detection algorithm for real-time data scanning while the sensors are in surveillance. The second is to mine (or scan) an archived surveillance video database in which all the video data are stored in a compressed format; the fast scanning capability allows efficiently automatic mining the data to retrieve the shots containing independent motion.

In order to achieve these objectives, the proposed approach is based on the linear system consistency analysis, and consequently is called LSCA. Since the LSCA approach only focuses on what exactly is necessary to compute, it saves the computation to a minimum and achieves the efficacy to the maximum. Technically, as compared with the existing techniques and available technologies, the LSCA method has the following distinctive advantages: (1) No camera calibration is required or necessary, i.e., image coordinates directly from the video frame may be used without having to convert them into calibrated coordinates. (2) The statistics computed in the algorithm are stable due to the Low condition numbers of the matrices, resulting in avoiding the unstable matrix computation problem of high condition numbers typically existing in many computer vision and image understanding techniques. (3) LSCA is able to detect independent motion only based on two frames, as opposed to some techniques in the literature requiring more than two frames. (4) LSCS complexity is very low, and is able to have a fast detection. (5) LSCA directly works on the compressed data; it does not need to decompress a video. (6) LSCA only requires one camera video stream in order to detect independent motion, as opposed to some techniques in the literature that require stereo video streams.

\section{LINEAR SYSTEM CONSISTENCY ANAL- YSIS}

We use a $3 \mathrm{D}$ to $2 \mathrm{D}$ affine model to approximate the video camera imaging system. For typical surveillance video, this affine model is sufficiently accurate to model the mapping from $3 \mathrm{D}$ scenes to $2 \mathrm{D}$ images. Our experiments also show that this model even works well for some of the non-surveillance video such as movies (see Fig. 6 for an example).

Given a $3 \mathrm{D}$ point $\boldsymbol{P}$ and its corresponding $2 \mathrm{D}$ point $\boldsymbol{p}$, a $3 \mathrm{D}$ to $2 \mathrm{D}$ affine transform is a linear transform, and is defined as [20]:

$$
p=A P+t
$$

where $\boldsymbol{A}$ is a 2 by 3 matrix with six independent parameters, and $\boldsymbol{t}$ is a $2 \mathrm{D}$ vector with another two independent parameters.

Assume that the camera motion between two arbitrary frames is an arbitrary 3D motion, which can be represented as a 3 by 3 rotation matrix $\boldsymbol{R}$ with three independent parameters, and a $3 \mathrm{D}$ translation vector $\boldsymbol{T}$ with another three independent parameters.

$$
\boldsymbol{P}^{\prime}=\boldsymbol{R P}+\boldsymbol{T}
$$

where $\boldsymbol{P}^{\prime}$ is the same point of $\boldsymbol{P}$ after the camera motion in the 3D space. The displacement of the point $\boldsymbol{P}$ in the 3D space after the motion is:

$$
\dot{\boldsymbol{P}}=\boldsymbol{P}^{\prime}-\boldsymbol{P}=(\boldsymbol{R}-\boldsymbol{I}) \boldsymbol{P}+\boldsymbol{T}
$$

where $\boldsymbol{I}$ is the identity matrix. From Eq. 1 and Eq. 3, it is clear:

$$
\dot{\boldsymbol{p}}=\boldsymbol{A P}=\boldsymbol{A}(\boldsymbol{R}-\boldsymbol{I}) \boldsymbol{P}+\boldsymbol{A T}
$$

Let $\boldsymbol{P}=(X, Y, Z)^{T}$ and $\boldsymbol{p}=(x, y)^{T}$. Given each image point $\boldsymbol{p}$, Eqs. 4 and 1 give rise to four independent equations. Eliminating $\boldsymbol{P}$, we obtain a linear constraint for each image point $\boldsymbol{p}$ in a video frame:

$$
\dot{x}+\theta \dot{y}+\alpha x+\beta y+\gamma=0
$$

where the variables $\alpha, \beta, \gamma$, and $\theta$ are functions of the motion parameters $\boldsymbol{R}, \boldsymbol{T}$ between the two frames, and the sensor parameters $\boldsymbol{A}, \boldsymbol{t}$ with the following relationship:

$$
\alpha=\frac{f\left(m_{i j}\right)}{g\left(m_{i j}\right)}
$$

where $m_{i j}$ are the motion parameters (the elements of $\boldsymbol{R}$ and $\boldsymbol{T})$ and/or the sensor parameters (the elements of $\boldsymbol{A}$ and $\boldsymbol{t}$ ), and $f, g$ are both quadratic functions. Similar expressions exist for $\beta, \theta, \gamma$.

When any two frames are determined, the motion parameters $\boldsymbol{R}, \boldsymbol{T}$ are constants for all the image points in the frames. We first assume that the sensor parameters $\boldsymbol{A}, \boldsymbol{t}$ are always constants. We will relax this assumption later. Hence, the variables $\alpha, \beta, \gamma$, and $\theta$ are independent of the image coordinates $x, y$, and their derivatives $\dot{x}, \dot{y}$ in Eq. 5 . This indicates that for each point in a frame, there is a linear constraint represented in Eq. 5.

Now assume that we have $n$ points identified in a frame, and that we know each point's displacement $(\dot{x}, \dot{y})^{T}$ to another frame. Since each point contributes one linear constraint of Eq. 5, we have a linear system consisting of these $n$ points:

$$
D \xi=b
$$




$$
\boldsymbol{D}=\left(\begin{array}{cccc}
1 & \dot{y_{1}} & x_{1} & y_{1} \\
\ldots & & & \ldots \\
1 & \dot{y_{n}} & x_{n} & y_{n}
\end{array}\right) \boldsymbol{\xi}=\left(\begin{array}{c}
\theta \\
\alpha \\
\beta \\
\gamma
\end{array}\right) \boldsymbol{b}=\left(\begin{array}{c}
-\dot{x_{1}} \\
\ldots \\
-\dot{x_{n}}
\end{array}\right)
$$

Given such a linear system, if we know that all the $n$ points are with the camera motion, i.e., there is no independent motion with any of these $n$ points, then all the $n$ points have the same motion parameters. Since the sensor parameters are always the same for all the points, the $n$ points will have the same values $\alpha, \beta, \theta, \gamma$ based on Eq. 6 . Therefore, the linear system Eq. 7 is consistent, i.e., there are solutions to this system. This has proven the following theorem:

TheOREM 3.1. Given $n$ points represented in the linear system in Eq. 7, if there is no independent motion with any of these points, then the linear system is consistent.

This means that the consistency of the linear system is the necessary condition of no independent motion in the $n$ points. In general, given $n>4$, the rank of $\boldsymbol{D}$ is 4 . Consequently, in general the consistency of Eq. 7 means there is a unique solution to this linear system.

From Theorem 3.1, it is clear that if the linear system Eq. 7 is not consistent, there must be independent motion involved. However, the linear consistency of the system Eq. 7 is not the sufficient condition for detecting any independent motion of the $n$ points. This is due to the fact that Eq. 6 is not a one to one mapping between a specific vector of $\boldsymbol{\xi}$ and a specific set of motion parameters. Given the same solution $\boldsymbol{\xi}$ for all the $n$ points, it is possible to have different values of motion parameters that satisfy the same mapping of Eq. 6. Fig. 2 illustrates one example of this situation. In this example, the camera moves from the position $\boldsymbol{O}$ at time $t_{1}$ to the position $\boldsymbol{O}^{\prime}$ at time $t_{2}$ with a different orientation. If there is a $3 \mathrm{D}$ point $\boldsymbol{Q}$ which does not involve any independent motion between $t_{1}$ and $t_{2}$, then the corresponding images of $\boldsymbol{Q}$ at these two instants are $\boldsymbol{p}$ and $\boldsymbol{q}$, respectively. Thus, the displacement between the two frames for $\boldsymbol{Q}$ in the image domain is $\boldsymbol{q}-\boldsymbol{p}$. Now as another scenario, if there is another 3D point $\boldsymbol{P}$ located somewhere in the ray between $\boldsymbol{Q}$ and $\boldsymbol{O}$ at time $t_{1}$, then the image of $\boldsymbol{P}$ is the same $\boldsymbol{p}$ as that of $\boldsymbol{Q}$. When the camera moves from $\boldsymbol{O}$ to $\boldsymbol{O}^{\prime}, \boldsymbol{P}$ undergoes an independent motion to $\boldsymbol{Q}$. Consequently, the combined motion of $\boldsymbol{P}$ results in the same displacement vector $\boldsymbol{q}-\boldsymbol{p}$ in the image domain. This means that given the same displacement vectors, together with the same points in one frame, subsequently resulting in the same $\boldsymbol{\xi}$ vector from Eq. 7, we cannot tell whether there is independent motion involved. Hence, we have disproved that the linear system consistency is the sufficient condition for detecting independent motion.

Though the consistency of the linear system Eq. 7 is only the necessary condition and not the sufficient condition to determine whether there is any independent motion involved in the $n$ point set, we can still use it to detect the indepenent motion. In fact, if the linear system is not consistent, we can immediately conclude that there is independent motion involved between the two frames. On the other hand, if the linear system is consistent, we may presume that there is no independent motion. This presumption is subject to a potential false negative, as the linear consistency is not the sufficient condition for the independent motion detection. Similarly, if a large computation noise occurs (e.g., the image point localization errors, the displacement vec-

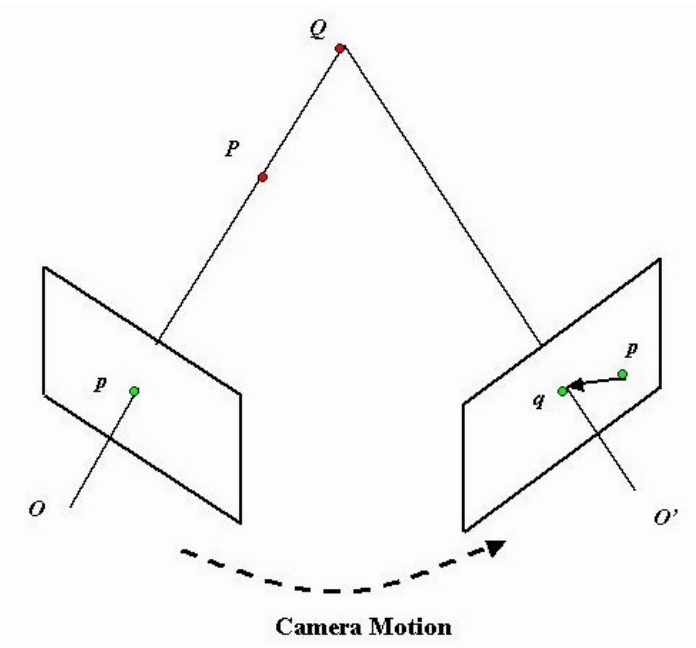

Figure 2: An example to show that the same displacement, which generates the same $\xi$ vector, is not sufficient to tell whether there is an independent motion.

tor estimation errors), a consistent linear system could turn out to be inconsistent. In this case, a false positive would be generated. In general, a few false positives are allowed while false negatives must be guaranteed to a minimum.

Now the question is, given $n$ points in two frames, how to determine whether the linear system Eq. 7 is consistent. By linear algebra theory [23], Eq. 7 is consistent iff

$$
\operatorname{Rank}(\boldsymbol{D})=\operatorname{Rank}(\boldsymbol{D} \boldsymbol{b})
$$

where $\boldsymbol{D} \boldsymbol{b}$ is the augmented matrix of Eq. 7. In order to determine the rank of the above two matrices, we apply singular value decomposition (SVD) to both $\boldsymbol{D}$ and $\boldsymbol{D} \boldsymbol{b}$, and define

$$
R=\frac{\sigma_{\min }(\boldsymbol{D})}{\sigma_{\min }(\boldsymbol{D b})}
$$

where $\sigma_{\min }(\boldsymbol{D})$ and $\sigma_{\min }(\boldsymbol{D} \boldsymbol{b})$ are the smallest singular values of $\boldsymbol{D}$ and $\boldsymbol{D} \boldsymbol{b}$, respectively, assuming Eq. 7 has unique solution if it is consistent; multiple solution cases may be handled similarly. Consequently, Eq. 7 is consistent iff $R$ is above a threshold, following the theory and practice of [46, $47,44]$. Note that from the definition of Eq. $9, R \geq 1$.

There are two points that are worth mentioning regarding the computation of the linear consistency of Eq. 7 .

- The transform Eq. 1 does not assume the calibration of the camera, allowing use of uncalibrated image coordinates $x_{i}, y_{i}$ in the linear system Eq. 7, and empowering this approach for practical applications, as in typical video data, the sensor parameters are unknown.

- The coefficients of the linear system Eq. 7 are all linear terms of $x_{i}, y_{i}, \dot{x}_{i}, \dot{y}_{i}$. This eliminates the popular but notorious problem of high condition numbers in linear systems typically existing in many image understanding solutions [17] that makes the solutions unstable. This property indicates that the $R$ statistic defined in Eq. 9 is stable, which is confirmed by the experiments.

\section{LSCA METHOD}


Two potential problems are revealed in the linear system Eq. 7. Eq. 7 assumes the availability of displacement vectors $(\dot{x}, \dot{y})^{T}$ for a group of image feature points in a frame. In order to obtain the displacement vectors, a correspondence algorithm or an optical flow algorithm must be applied, such as $[26,37,48]$. Since the correspondence or the flow computation problem [2] is an ill-posed problem in computer vision and image understanding research, false positives and false negatives in terms of the correspondence errors between two frames are inevitable, which will be propagated to false positives and false negatives in independent motion detection. Consequently, it is desirable to have a linear system independent of any specific correspondence algorithms or optical flow algorithms for computing the displacement vectors.

The second problem is that the current linear system Eq. 7 assumes the availability of two static images, as opposed to two video frames. Video frames are typically stored in compressed data based on a specific video format such as MPEG, unless they are explicitly decompressed to become static images. Therefore, it is also expected to have a linear system directly based on compressed video data. Below we propose a solution to avoiding the two problems leading to the LSCA method based on the MPEG standard.

Recall Eqs. 1 and 4. Instead of applying them to a set of feature points in a frame, we now apply them to every points of a region with $m$ points in the frame. Thus, we have

$$
\begin{gathered}
\sum_{i=1}^{m} \dot{\boldsymbol{p}}_{i}=\boldsymbol{A}(\boldsymbol{R}-\boldsymbol{I}) \sum_{i=1}^{m} \boldsymbol{P}_{i}+m \boldsymbol{A} \boldsymbol{T} \\
\sum_{i=1}^{m} \boldsymbol{p}_{i}=\boldsymbol{A} \sum_{i=1}^{m} \boldsymbol{P}_{i}+m \boldsymbol{t}
\end{gathered}
$$

Define

$$
\begin{gathered}
\overline{\boldsymbol{p}}=\frac{1}{m} \sum_{i=1}^{m} \boldsymbol{p}_{i}=(\bar{x}, \bar{y})^{T} \\
\overline{\dot{\boldsymbol{p}}}=\frac{1}{m} \sum_{i=1}^{m} \dot{\boldsymbol{p}}_{i}=(\overline{\dot{x}}, \overline{\dot{y}})^{T} \\
\overline{\boldsymbol{P}}=\frac{1}{m} \sum_{i=1}^{m} \boldsymbol{P}_{i}=(\bar{X}, \bar{Y}, \bar{Z})^{T}
\end{gathered}
$$

we obtain

$$
\begin{gathered}
\overline{\dot{p}}=\boldsymbol{A}(\boldsymbol{R}-\boldsymbol{I}) \overline{\boldsymbol{P}}+\boldsymbol{A T} \\
\overline{\boldsymbol{p}}=\boldsymbol{A} \overline{\boldsymbol{P}}+\boldsymbol{t}
\end{gathered}
$$

If we take each MPEG macroblock as such a region, then $m$ becomes a constant (i.e., $m=256$ ) over the whole frame. Therefore, we have a similar linear constraint for each macroblock of a frame:

$$
\overline{\dot{x}}+\theta \overline{\dot{y}}+\alpha \bar{x}+\beta \bar{y}+\gamma=0
$$

and consequently, given $n$ macroblocks, we can build a similar linear system

$$
D_{m}=\xi_{m} b_{m}
$$

with a similar theorem:
THEOREM 4.1. Given $n$ macroblocks in an MPEG video frame represented in the linear system in Eq. 15, if there is no independent motion with any of these macroblocks, then the linear system is consistent.

In the MPEG compression standard, for each macroblock in a frame, if this macroblock is inter-coded, there is a motion vector available. We approximate $\overline{\dot{p}}$ with the motion vector, and $\overline{\boldsymbol{p}}$ is the center of the macroblock. Since the macroblock information (including the motion vector and the center coordinates) can be easily obtained directly from a compressed MPEG video stream, we have a linear system Eq. 15 that can directly work on the MPEG compressed data without having to depend on a specific algorithm to compute the correspondence or optical flow between the two frames, simultaneously eliminating the two potential problems mentioned above with Eq. 7. If the macroblock is intra-coded, we just exclude this macroblock from the linear system of Eq. 15. If the frame is an I frame in which all the macroblocks are intra-coded, from Eqs. 14, 15, and 9, $R=1$. This could be a false positive, which can be easily removed by filtering the $R$ statistics, resulting in rejection of this false positive in the final detection.

While the displacement vector may be approximated by the motion vector of a macroblock, this may create another problem, i.e., how accurate this approximation is. It is known [39] that the motion vector estimation in MPEG is subject to errors, and how large the errors are depends on the specific implementation of the motion vector estimation algorithm under the MPEG standard [8]. The accurate relationship between the errors in motion vector estimation in MPEG and the detection accuracy requires further study. Here we provide a tentative solution to this problem based on the normal flow computation to attempt to lower the potential errors for the motion estimation. Research shows $[43,35,25]$ that the normal flow is more reliable than standard optical flow. Assuming that the intensity function of a frame is $I(x, y)$, the normal flow $n_{p}$ at the point $\boldsymbol{p}=(x, y)^{T}$ is defined as the dot product between the gradient of the point $\boldsymbol{p}$ and the displacement vector at this point:

$$
n_{p}=\frac{\partial I}{\partial x} \dot{x}+\frac{\partial I}{\partial y} \dot{y}
$$

Since in the compressed MPEG video stream we only have the motion vectors for each macroblocks as opposed to each points, we must extend this point-based normal flow definition to the macroblock based one. Let $\nabla I(\boldsymbol{p})$ be the gradient of the intensity function $I$ at a point $\boldsymbol{p}$. Given a macroblock $\boldsymbol{M}$, the macroblock gradient $\nabla I(\boldsymbol{M})$ is defined as:

$$
\nabla I(\boldsymbol{M})=\frac{1}{m} \sum_{i=1}^{m} \nabla I\left(\boldsymbol{p}_{i}\right)
$$

where $\boldsymbol{p}_{i}$ is a point of $\boldsymbol{M}$, and $m$ is the total number of points in $\boldsymbol{M}$. In MPEG, $m=256$.

Now the question is how to estimate the gradient of a macroblock without decompressing the video data. Lee et al [24] showed a method of estimating the approximated gradient for a whole block only using a few low frequency AC coefficients of the DCT of the block in MPEG. This is essentially to approximate the original DCT AC coefficients 
$A C_{u v}$ with the corresponding "continuous" versions $A \tilde{C}_{u v}$ :

$$
A C_{u v} \approx A \tilde{C}_{u v}=C(u) C(v) \int_{0}^{8} \int_{0}^{8} \cos \frac{u \pi}{8} \cos \frac{v \pi}{8} f(x, y) d x d y
$$

where $C(u)$ and $C(v)$ are the scale factors of the standard DCT definition [39]. Given a few limited lower terms of $A C_{u v}$, we can explicitly solve for the block edge orientation, the block edge offset, and the block edge strength [24]. The question, however, is how many such lower AC coefficients would suffice an accurate estimate of the block gradient. Reported research [24] shows that in order to estimate the block gradient, only the five low frequency $\mathrm{AC}$ coefficients are necessary to recover the information (i.e., $\left.A C_{01}, A C_{10}, A C_{20}, A C_{11}, A C_{02}\right)$. Consequently, the majority of the AC coefficients as well as the DC component are not required. This shows that it is still not necessary to decompress the video stream in order to recover the block gradient; the method can directly work on the compressed MPEG stream to extract the small piece of the "essential" information (i.e., the motion vector of a macroblock and the five low frequency AC components of a block) without having to decompress the video stream. Fig. 4 (a) - (c) shows examples of the "block" edges detected based on estimating the block gradients using the five lowest $\mathrm{AC}$ components of the blocks. Note that due to the background noise (e.g., the different background objects in Fig. 4 (a) and (b)), "edge" information is indicated through the block gradient detection; when the background is noise-free (e.g., in Fig. 4 (c)), there is no such "edge" information.

Once we have the block gradient vectors available for all the four blocks of a macroblock, the macroblock gradient is computed by averaging the four block gradient vectors based on the definition. Finally, the normal flow value of a macroblock, $n(\boldsymbol{M})$, is defined similar to that of a point in Eq. 16 by taking the dot product between the macroblock gradient vector, $\nabla I(\boldsymbol{M})$, and the motion vector of this macroblock, $V(\vec{M})$

$$
n(\boldsymbol{M})=\nabla I(\boldsymbol{M}) \cdot V(\overrightarrow{\boldsymbol{M}})
$$

When we have the normal flow value computed for a macroblock, we can make a decision about whether this macroblock should be incorporated into the linear system of Eq. 15. The rationale [43] is that if the normal flow is low, the motion vector is probably not accurately estimated; consequently this macroblock should be rejected from incorporating into Eq. 15.

Now the LSCA algorithm is summarized as follows, which takes four parameters: the normal flow threshold $T_{n}$, the scan window width $r$, the $R$ statistic threshold $T_{R}$, and the defined minimum number of frames $T_{f}$ of a segment that contains independent motion.

Scan an input video stream in compressed MPEG

For every pair of consecutive frames

Start to build up the linear system Eq. 15

For each macroblock $M$ of the first frame $l$ of the pair Estimate the normal flow $n(\boldsymbol{M})$ of $\boldsymbol{M}$

If $n(M)>T_{n}$

Incorporate $\boldsymbol{M}$ into Eq. 15 based on Eq. 14

Compute $R$ of the linear system Eq. 15

Compute the median filtered $\bar{R}$ over a window of $r$ frames If $\bar{R}-1>T_{R}$
Label $l$ as no IM (NIM)

Else, label $l$ as a frame with IM (IM)

Any IM segment with frame number $>T_{f}$ is retrieved

Note that LSCA is based on the assumption of constant camera model in terms of the sensor parameters $\boldsymbol{A}$ and $\boldsymbol{t}$. In real applications, it is possible that the sensor parameters change during the surveillance (e.g., zoom in/out). Since LSCA only focuses on two consecutive frames, given the current video frame rate (about 30 frames/second), if the change is slow, we can ignore the change and still use the algorithm to compute the $R$ statistic between two frames; if the change is fast, the computed $R$ value between two frames could be wrong, which would lead to a false positive or negative. However, in this case, there will be only a few frames subject to the error of $R$ values, and they will be shown as outliers and will then typically be filtered out by LSCA (see Fig. 5 for an example).

\section{EXPERIMENTAL EVALUATIONS}

In this section, we first report a preliminary, simulation based analysis on estimating the detection false positives and false negatives, as well as the detectability bounds. We then present the real data experimental evaluations to demonstrate the robustness and effectiveness of LSCA.

\subsection{Simulation Analysis}

Both analyses on false positives and false negatives are the sensitivity analysis for LSCA. This may be achieved by testing the stability of the statistic $R$ of LSCA under different levels of noise through simulation. As a preliminary analysis, we design a simulation scenario as follows.

$103 \mathrm{D}$ points are randomly generated in a Euclidean world coordinate system. A video camera is first located at an arbitrary position in this world coordinate system. The 10 points are projected to the image plane of the camera based on the $3 \mathrm{D}$ to $2 \mathrm{D}$ affine transform to generate the image coordinates in this frame. Then the camera is moved to another position, and the $103 \mathrm{D}$ points are projected to the image plane again to generate the image coordinates in the second frame. The image displacement vectors are immediately obtained from the image coordinates of the two frames. The displacement vectors and the image coordinates in the first frame are then corrupted by different levels of randomly generated Gaussian noise, parameterized by different deviations in terms of the number of pixels. The image points in the two frames are distributed in an area of 100 pixels by 120 pixels. Thus, 1 pixel deviation of Gaussian noise approximately corresponds to $1 \%$ of the whole effective image dimension.

The corrupted image coordinates and the displacement vectors are input into LSCA, and the $R$ statistic is computed for each noise level. Since there is no independent motion involved in this scenario, the $R$ value should be high. Under the corruption of the noise, however, the $R$ value degrades as the noise level increases. Fig. 4(a) shows the logarithm of the $R$ values averaged over 1000 runs with different seeds under each Gaussian noise level parameterized by standard deviation in terms of the number of pixels.

From Fig. 4(a), it is clear that false positives of independent motion detection may occur when the noise level increases. From the Figure, if the noise level is controlled 

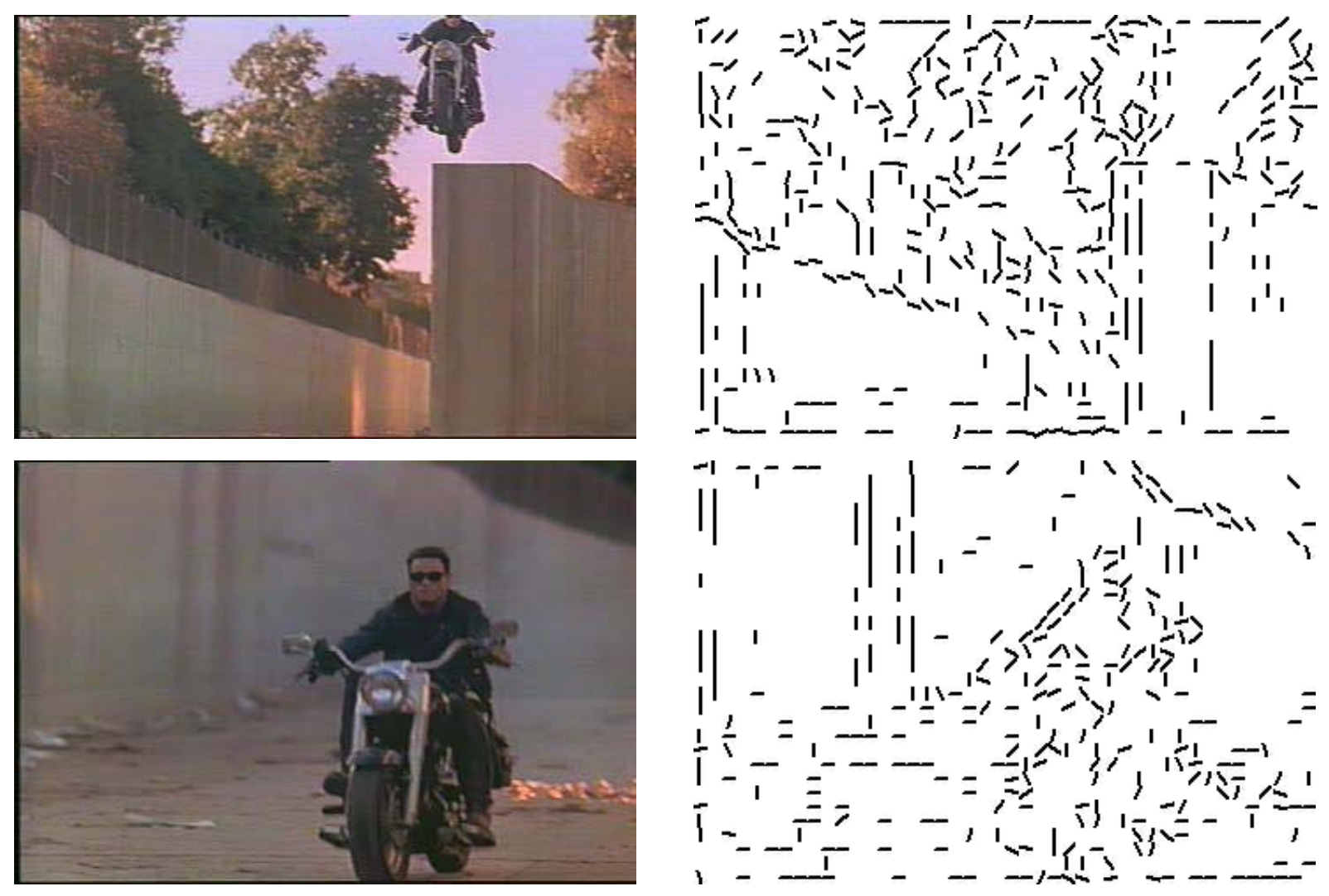

(a)
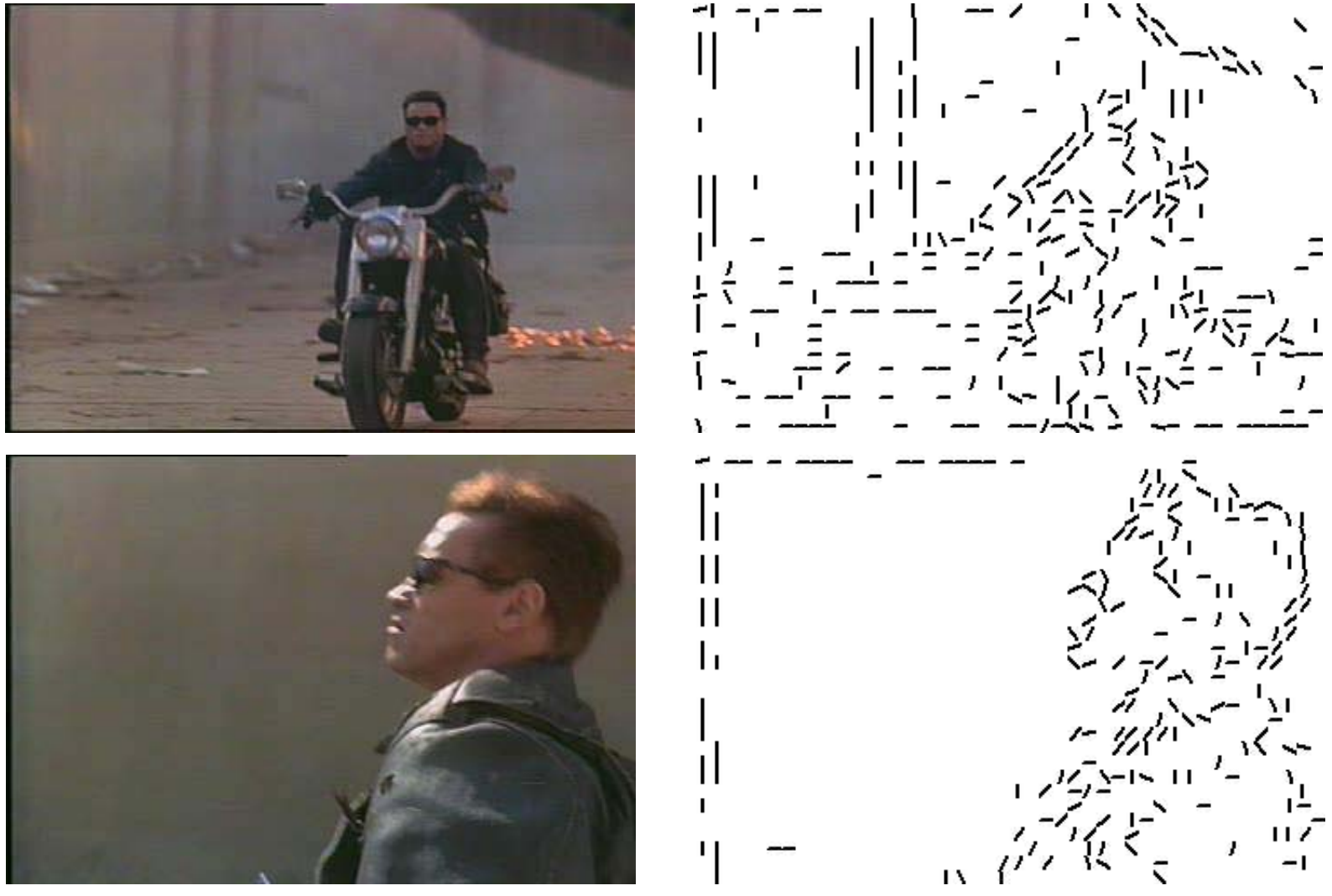

Figure 3: Examples of using the five lowest DCT AC coefficients in each block to estimate the gradient information, where the left column shows the frame samples while the right column shows the corresponding block edge maps. Note that no decompression is performed. 
under 2 pixels, the $R$ value always stabilizes somewhere statistically significantly higher than 1 (above 2 ). Note that considering the effective image dimension as 100 by 120,2 pixels' noise is significantly large in practice. This shows that LSCA is very robust in rejecting false positives in independent motion detection.

The simulation scenario continues when a point of independent motion is added into the original 10 point set. This time the $R$ value always stays at 1 regardless of what level the noise is, indicating LSCA is effective in detecting independent motion.

While false positives and false negatives are the probabilities describing an event of a detection failure of the LSCA, detectability is an issue of how significant an independent motion should be such that LSCA is able to detect it. Detectability is a different but a related concept, which is defined as the smallest independent motion that LSCA can detect. Again, this may be determined through simulation analysis.

Using the same simulation above, based on the original 10 background points, we add the 11th point that is subject to an independent motion, in addition to the camera motion. In order to separate contributions from different independent motion components to the detectability of LSCA, we apply LSCA to independent translations of this point along $X, Y, Z$ axes, and to independent rotations of this point about $X, Y, Z$ axes, respectively, under different levels of Gaussian noise. Fig. $4(\mathrm{~b})$ to $(\mathrm{g})$ show the six scenarios of the simulation.

A qualitative examination of these simulation results reveals that the performance of LSCA appears less sensitive to the independent motion related to the $Z$ axis (rotation about the axis or translation along the axis) than to the independent motion related to the other axes; quantitatively, based on this simulation, the detectability is related to the noise levels, and a higher noise level increases detectability. The reason is that a higher level of noise increases the false positives, which help increase detectability. Take the noise level of 1.5 pixel deviation for example. If the threshold value is set as 2 for $R$, the detectability is under 1 unit for all the translations, and under 0.1 degree for all the rotations. If the threshold of $R$ decreases to 1.5 , the detectability for translations along $\mathrm{X}, \mathrm{Y}$, and $\mathrm{Z}$ axes is above 8,10 , and 20 units, respectively, for rotations about $\mathrm{X}, \mathrm{Y}$, and $\mathrm{Z}$ axes is above $0.4,0.8$, and 3.0 degrees, respectively. Note that these parameters are obtained from this specific set of simulation only. More accurate parameters will be determined based on a larger scale of simulation as part of the future work.

\subsection{Real Data Evaluations}

We have implemented the LSCA as a stand alone version in a Windows2000 platform with Pentium III $800 \mathrm{MHz}$ CPU and 512 MB memory. Fig. 1(c) and (d) show the original $R$ statistics computed at every frames for the two shots from two surveillance videos in Fig. 1(a) and (b), respectively. The statistics are obvious to tell whether and where there is independent motion in the video. The first shot containing 1119 frames describes an independent motion of a missile launcher moving to its destination. The mean of the original $R$ is 1.0 and the deviation is 0.00122 over the 1119 frames. The second shot containing 1058 frames surveys an area of ground terrain with no independent motion. The mean of the original $R$ is 1.389 and the deviation is 0.169 over the 1058 frames.

Fig. 5 shows the original $R$ statistics in another very noisy surveillance video that contains scenarios of both independent motion and no independent motion. There are a noticeable number of outliers scattered over the 4564 frames of this video. Some of them are due to camera parameter change (e.g., zoom in/out) during the surveillance; others are false positives or false negatives, caused by the poor estimation of the motion vectors due to the poor image quality of the video, or even no motion vector such as in an I frame. However, almost all of these outliers are removed after the median filtering in the LSCA, resulting in a very robust detection of independent motion.

In order to give a meaningful evaluation, we make an assumption that a reliable independent motion shot should last at least 30 frames, which corresponds at least about one second presence of independent motion in the video. This assumption ensures that any sporadic detection false positives due to motion estimation outliers and/or sensor parameter changes will be removed. Since LSCA performs frame-based independent motion detection, it is reasonable to define the detection precision as the percentage of the number of truthed independent motion frames detected by LSCA of the total number of detected independent motion frames, and to define the detection recall as the percentage of the number of truthed independent motion frames detected by LSCA of the total number of truthed independent motion frames in a video. We have applied LSCA to a collection of manually truthed surveillance video data of over one and half hours with over 160,000 frames in total as an objective evaluation. The precision is $81.27 \%$ and the recall is $93.6 \%$. Therefore, the false positives are $18.73 \%$ and the false negatives are $6.4 \%$ in this evaluation. The low false negatives show that LSCA is a conservative approach to mining independent motion in surveillance video. The frames that LSCA fails to detect are those with small independent motion. The future work of this project will address the detectability issue more extensively. The majority of the false positives are due to poor motion estimation in MPEG, possibly due to poor image quality (some of them are night video with low visibility). The rest of the false positives are due to camera parameter changes (longer than 30 frames). The future work will focus on more robustly filtering out outliers using a technique similar to least median squares [45] or RANSAC [14].

To show that LSCA is not only valid for the typical surveillance scenario where the camera is far away from the scene, but also valid for the scenario where the camera is relatively close to the scene, Fig. 6 demonstrates an experimental result of LSCA in which we take a movie with an independent motion very close to the camera, and split the spatial domain into the left and the right halves such that the left video does not contain independent motion while the right one does. The result clearly shows that LSCA is robust even under the situation where the camera is close to the scene.

Since LSCA essentially just needs to compute the $R$ value for each frame, and since in each frame there is typically a very limited number of macroblocks, the complexity of LSCA is very low. The current prototype of LSCA scans a compressed MPEG video with a typical frame resolution of 240 by 350 at the speed of 35 frames/second under the 


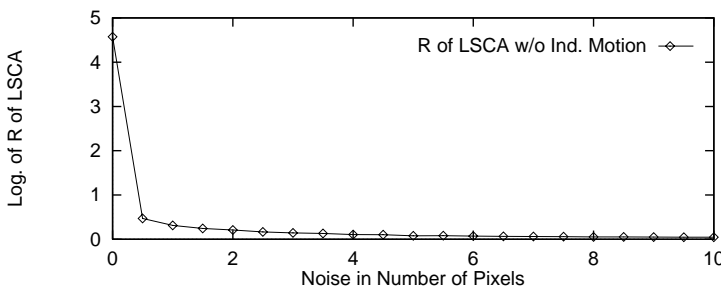

(a)
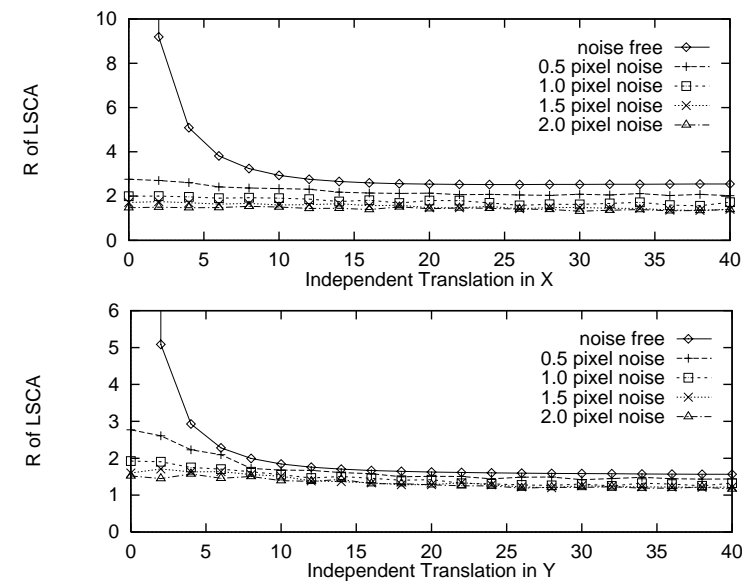

(b)

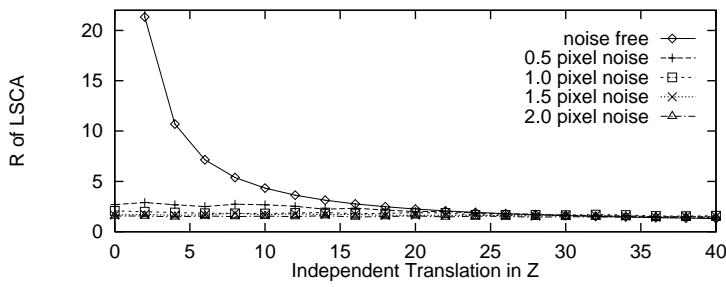

(c)

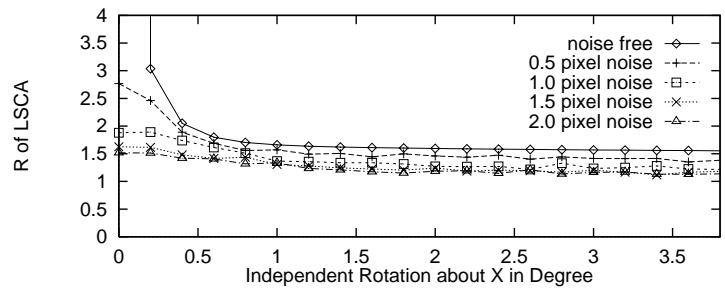

(d)

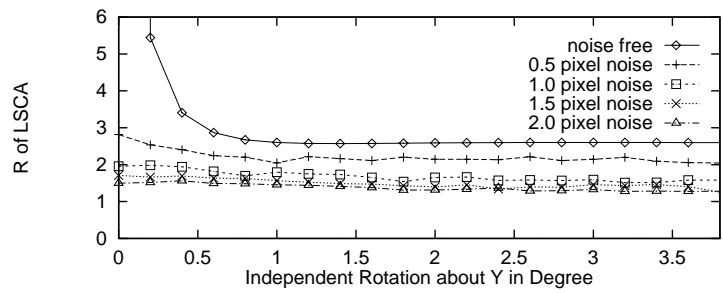

(e)

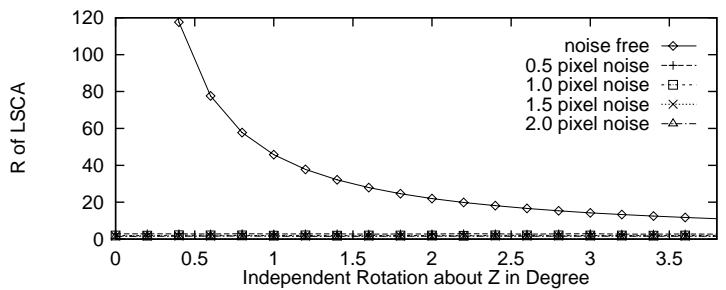

(f)

(g)

Figure 4: (a) Logarithm of the $R$ statistic of LSCA under different Gaussian noise levels when no independent motion involved. (b)-(d) Detectabilities of LSCA w.r.t. independent translations along $X, Y$, and $Z$ axes, respectively. (e)-(g) Detectabilities of LSCA w.r.t. independent rotations about $X, Y$, and $Z$ axes, respectively. 


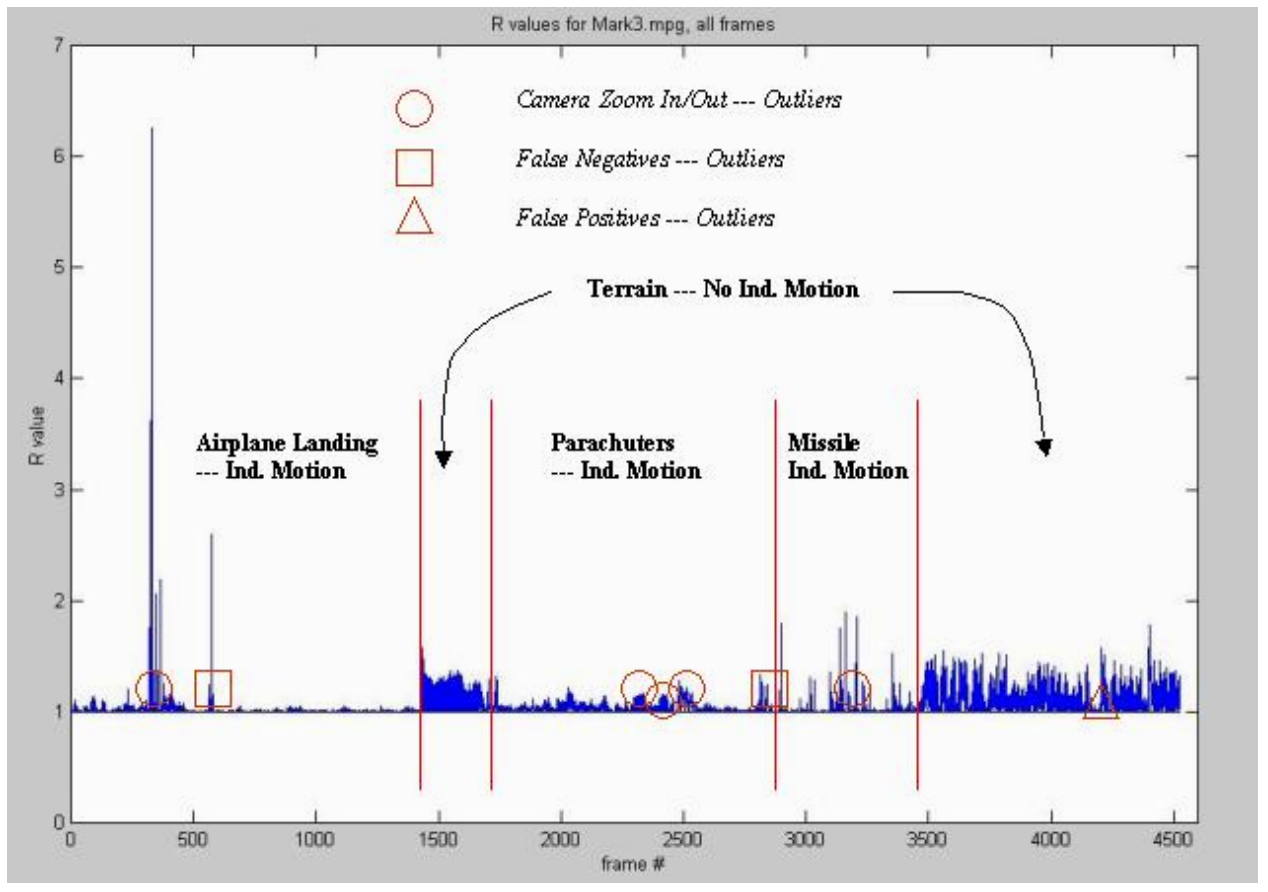

Figure 5: The original $R$ statistics computed for a whole video containing 4564 frames in total before the median filtering of LSCA is applied; after the filtering, almost all of the outliers are rejected.

current platform, which is faster than real-time. Note that this implementation is just for proof of the concept and the code has not been optimized yet. This shows that LSCA holds a great promise and vitality in the future applications in both proposed scenarios: real time surveillance data scanning equipped with the sensors and fast data mining for an ahchived database of surveillance data.

\section{SUMMARY OF THE ADVANTAGES}

Based on the analysis given above, it is clear that LSCA has the following distinctive advantages as compared with the existing methods in the literature:

- No camera calibration is required or necessary in order to apply LSCA, i.e., the uncalibrated image coordinates directly from the video frame may be used without having to convert them into the calibrated coordinates.

- The statistics computed in LSCA are stable due to the low condition number in the linear system, resulting in avoiding the unstable matrix computation problem of high condition numbers typically existing in many computer vision and image understanding techniques.

- LSCA is able to detect independent motion only based on two frames, as opposed to more than two frames required by some techniques in the literature (e.g., the methods by Irani and Anandan [19] and by Sawhney et al [34] require three frames). Those that require more than two frames typically attempt to do spatial identification of the specific independently moving targets in the frames.

- LSCA is very fast. The current prototype with evennot-optimized-yet implementation runs at 35 frames/second detection accuracy. rate for a typical frame resolution of $240 \times 350$ of compressed MPEG videos on a Pentium III $800 \mathrm{MHz}$ Windows 2000 system with 512 MB memory.

- LSCA directly works on the compressed data without having to decompress the data first.

- LSCA only requires one camera video stream for robust detection as opposed to the stereo video streams required by some techniques in the literature (e.g., the techniques proposed by Argyros et al [5, 3, 4, 6] and by Lourakis et al [25]).

\section{CONCLUSIONS AND DISCUSSIONS OF THE FUTURE WORK}

This paper presents a novel solution to the problem frequently encountered in military/intelligence surveillance: automatic mining video data for independently moving targets. Instead of attempting to detect each individual independently moving target in each frame, this method focuses on determining whether or not each frame has independent motion. Consequently, it differs from the existing literature on this topic in the following two aspects: (1) fast detection (2) detection directly from the compressed video streams instead of from the image sequences. These two aspects are motivated from the applications that this research aims to address, and therefore, are the contributions to the literature of independent motion detection. The solution is based on the Linear System Consistency Analysis, and thus is called LSCA. A number of distinctive technical advantages of LSCA as compared with the existing methods in the literature are identified. Evaluations from both simulated and real data show that LSCA holds a great promise in achieving fast detection without sacrificing the reasonable 
As have been identified during the current research, the following problems will be targeted as the future work to further the feasibility research for LSCA in real surveillance applications:

1. Systematically determine the probabilities of false positives and false negatives, as well as the bounds of the detectability of LSCA.

2. Systematically determine the relationship between the errors in motion vector estimation in MPEG and the false positives and false negatives of LSCA, and based on this relationship, develop a more effective method for robust estimation of the $R$ statistic without sacrificing the already achieved efficient performance.

3. Extensively evaluate LSCA in a larger scale data testbed to report the final evalution results in the literature.

The false negatives analysis is related to the theoretic investigation of the sufficient condition of the independent motion detection based on the linear system approach. Specifically, the mapping relationship between $\boldsymbol{\xi}$ and the motion parameters $\boldsymbol{R}$ and $\boldsymbol{T}$ will be further analysed to predict a probability of the false negatives. This probability will be further verified through statistical simulation analysis.

The false positives analysis is related to the investigation of the errors in motion vector estimation and how the errors propagate to generate the false positives. This is related to the issue of the stability of the $R$ statistic estimation. Matrix computation theory will be applied to attempt to derive a theoretic bound on the probability of the false positives. Again simulation based statistical analysis will be performed to verify the bound.

In terms of the stability of the statistic $R$ analysis, we first aim at developing the error model of the motion vector estimation as its relationship to the stability of the statistic $R$. Similar to the approaches taken in the probability analysis for the fasle positives and the false negatives of LSCA, here we will also take two approaches simultaneously. The first approach is based on the theoretic analysis using matrix computation theory. The motion vector estimation error is propagated to the estimation error of $R$ through the singular value decomposition (SVD) of the coefficient matrix $\boldsymbol{D}_{\boldsymbol{m}}$ and the augmented matrix $\boldsymbol{D}_{\boldsymbol{m}} \boldsymbol{b}_{\boldsymbol{m}}$ in Eq. 15. This is essentially a problem that requires a solution that combines the general theory of SVD computation stability [16, 31] and the special advantages we can take of from the observed error patterns of the motion vector estimation in $\boldsymbol{D}_{\boldsymbol{m}}$ and $D_{m} b_{m}$.

The second approach is based on simulation to determine the error model and the relationship. The simulation will be performed similar to the one in the probability analysis for the detection false positives and false negatives. However, here the focus is on the noise of the displacement vector $(\overline{\dot{x}}, \overline{\dot{y}})$ and the $R$. The consistency issue between the results obtained from the two approaches will be analysed.

Finally, we will study how to maximally stabilize the $R$ statistic given the motion vector estimation error based on the developed error model. We plan to take two approaches simultaneously to ensure a robust detection of the independent motion based on the stabilized $R$ statistic: the global approach and the local approach. The local approach focuses on the current frame along with its immediately neighboring frames to obtain a stabilized $R$ statistic. The global approach makes the first scan of the video to obtain all the $R$ statistics for all the frames based on the local approach, and then make the decision of detecting all the independent motion in the video based on a "revised" overall $R$ statistic distribution over the video.

Specific local approaches depend on the specific error models to be determined and used. There are several possible approaches. If the error model is random, we can use robust statistical regression methods [28] such as RANSAC $[14,45]$ and the Least Median of Squares [32]. These methods essentially combinatorially take a subsystem of Eq. 15 and compute the corresponding $R$ till an $R$ statistic is stabilized. Some of these methods can handle very high breaking points. The advantage of these methods is that they are very robust towards outliers. The disadvantage is that the combinatorial search is expensive. However, considering that there is typically a very limited number of macroblocks in a frame of the video, this combinatorial search could be affordable. If necessary, we can set up a search limit bound to make the complexity tractable.

If the motion error modal is more systematically distributed, we can take a probabilistic approach. Based on the available simulation and evaluation data, we can estimate all the prior and conditional probabilities for the contributions from different possible error sources identified. The actual $R$ statistic in each frame may be determined as an expected value based on the Bayesian analysis. The advantage of this approach is its efficiency, which can still keep up the efficient performance. The disadvantage is that the estimation of all the probabilities could be subject to errors and might be biased towards the training data.

The global approach will essentially "reshape" the statistics of $R$, and therefore it requires context information from immediate and distant neighboring windows for each frame's $R$. Certain non-linear filters will be applied to this approach, and thus, it will delay the decision of the detection till it has scanned the whole video. Consequently, the real time, online detection capability will be compromised if this approach is taken. The advantage is that the detection will be more accurate and robust.

When the above two issues are resolved, LSCA is ready for extensive evaluations. The purposes of this larger scale evaluation are: (i) to have a chance to refine the parameters, to update the developed models, and to revise the implementation details in case there is anything found inconsistent with the algorithm parameters designed in the current version and used in the larger scale evaluations, and (ii) to obtain the final report of the detection accuracy in terms of the false positives and false negatives through the real data evaluation, and ensure that they are consistent with the ones obtained through the theoretic and simulation analyses. We will evaluate LSCA using the surveillance data not only obtained from aerial cameras in which the $3 \mathrm{D}$ to $2 \mathrm{D}$ affine model is valid, but also obtained from those cameras close to the scene in order to determine how far the affine camera model assumed in LSCA can scale up in keeping a good detection capability. LSCA might need to be minorly revised in certain details and parameter settings during these evaluations, and finally, the evaluation results will be reported.

\section{ACKNOWLEDGEMENTS}

This research is supported in part by AFOSR through 
the grant F49620-03-1-0007. The authors would like to acknowledge the technical advice kindly provided by Mark A. Robertson and Richard Simard at AFRL/IFEC. Xunyin Wang and Haroon Khan at SUNY Binghamton helped in part of the implementation and experiments in this project.

\section{REFERENCES}

[1] G. Adiv. Determining 3D motion and structure from optical flows generated by several moving objects. IEEE Trans. Pattern Analysis and Machine Intelligence, 7(4):384-401, 1985.

[2] P. Anandan. A computational framework and an algorithm for the measurement of visual motion. IJCV , 2, 1989.

[3] A. Argyros, M. Lourakis, P. Trahanias, and S. Orphanoudakis. Fast visual detection of changes in 3D motion. In Proc. IAPR Workshop on Machine Vision Applications, 1996.

[4] A. Argyros, M. Lourakis, P. Trahanias, and S. Orphanoudakis. Independent 3D motion detection through robust regression in depth layers. In Proc. British Machine Vision Conference, 1996.

[5] A. Argyros, M. Lourakis, P. Trahanias, and S. Orphanoudakis. Qualitative detection of 3D motion discontinuities. In Proc. IEEE/RSJ International Conference on Intelligent Robots and Systems, 1996.

[6] A. Argyros and S. Orphanoudakis. Independent 3D motion detection based on depth elimination in normal flow fields. In Proc. International Conference on Computer Vision and Pattern Recognition. IEEE Computer Society Press, 1997.

[7] S. Ayer, P. Schroeter, and J. Bigun. Segmentation of moving objects by robust motion parameter estimation over multiple frames. In Proc. European Conference on Computer Vision, 1994.

[8] http://mpeg.telecomitalialab.com/.

[9] P. Bouthemy and E. Francois. Motion segmentation and qualitative dynamic scene analysis from an image sequence. International Journal of Computer Vision, 10(2):157-182, 1993.

[10] Q. Cai and J. Aggarwal. Tracking human motion in structured environments using a distributed-camera system. IEEE Trans. Pattern Analysis and Machine Intelligence, 21(11):1241-1247, 1999.

[11] R. Cutler and L. Davis. Robust real-time periodic motion detection, analysis, and applications. IEEE Trans. Pattern Analysis and Machine Intelligence, 22(8):781-796, 2000.

[12] O. Faugeras. Three-Dimensional Computer Vision: A Geometric Viewpoint. MIT Press, 1993.

[13] S. Fejes and L. Davis. What can projections of flow fields tell us about the visual motion. In Proc. International Conf. Computer Vision, 1998.

[14] M. Fischler and R. Bolles. Random sample consensus: a paradigm for model fitting with applications to image analysis and automated cartography. Communications of $A C M, 24(6): 381-395,1981$.

[15] D. Forsyth, J. L. Mundy, A. Zisserman, C. Coelho, A. Heller, and C. Rothwell. Invariant descriptors for 3-D object recognition and pose. IEEE Trans. Pattern Analysis and Machine Intelligence, 13(10):971-991, 1991.
[16] G. Golub and C. Loan. Matrix Computations, 2nd Ed. The Johns Hopkins University Press, 1989.

[17] R. Hartley. In defence of the 8-point algorithm. In Proc. ICCV. IEEE, 1995.

[18] T. Huang and C. Lee. Motion and structure from orthographic views. IEEE Trans. Pattern Analysis and Machine Intelligence, 11:536-540, 1989.

[19] M. Irani and P. Anandan. A unified approach to moving object detection in $2 \mathrm{D}$ and $3 \mathrm{D}$ scenes. In Proc. of IUW, 1996.

[20] D. Jacobs. Recognizing 3-D Objects Using 2-D Images. Ph.D. Dissertation, MIT AI Lab., 1992.

[21] R. Jain. Segmentation of frame sequences obtained by a moving observer. IEEE Trans. Pattern Analysis and Machine Intelligence, 7(5):624-629, 1984.

[22] R. Kumar, P. Anandan, and K. Hanna. Direct recovery of shape from multiple views: a parallax based approach. In Proc. International Conf. Pattern Recognition, 1994.

[23] S. Lang. Linear Algebra, 3rd Ed. Springer-Verlag, 1987.

[24] S.-W. Lee, Y.-M. Kim, and S. Choi. Fast scene change detection using direct feature extraction from MPEG compressed videos. IEEE Trans. Multimedia, 2(4):240-254, 2000.

[25] M. Lourakis, A. Argyros, and S. Orphanoudakis. Independent 3D motion detection using residual parallax normal flow fields. In Proc. International Conference on Computer Vision. IEEE Computer Society Press, 1998.

[26] B. Lucas and T. Kanade. An iterative image registration technique with an application to stereo vision. In Proc. DARPA Image Understanding Workshop, 1981.

[27] S. Maybank. Theory of Reconstruction from Image Motion. Springer-Verlag, 1993.

[28] P. Meer, A. Mintz, and A. Rosenfeld. Robust regression methods for computer vision: a review. International Journal of Computer Vision, 6(1):59-70, 1991.

[29] R. Nelson. Qualitative detection of motion by a moving observer. In Proc. of CVPR. IEEE, 1991.

[30] R. Pless, T. Brodsky, and Y. Aloimonos. Detecting independent motion: the statistics of temporal continuity. IEEE Trans. Pattern Analysis and Machine Intelligence, 22(8):768-773, 2000.

[31] W. Press, S. Teukolsky, W. Vetterling, and B. Flannery. Numerical Recipes in C. Cambridge University Press, 1992.

[32] P. Rousseeuw and A. Leroy. Robust Regression and Outlier Detection. John Wiley and Sons, Inc., 1987.

[33] H. Sawhney. 3D geometry from planar parallax. In Proc. International Conference on Computer Vision and Pattern Recognition, 1994.

[34] H. Sawhney, Y. Guo, and R. Kumar. Independent motion detection in 3D scenes. IEEE Trans. Pattern Analysis and Machine Intelligence, 22(10):1191-1199, 2000.

[35] R. Sharma and Y. Aloimonos. Early detection of independent motion from active control of normal image flow patterns. IEEE Trans. SMC, 26(1):42-53, 
1996.

[36] A. Shashua and N. Navab. Relative affine structure: theory and application to 3D reconstruction from perspective views. In Proc. International Conference on Computer Vision and Pattern Recognition, 1994.

[37] J. Shi and C. Tomasi. Good features to track. In Proc. CVPR. IEEE, 1994.

[38] S. Smith and J. Brady. ASSET-2: real time motion segmentation and shape tracking. IEEE Trans. Pattern Analysis and Machine Intelligence, 17(8), 1995.

[39] A. Tekalp. Digital Video Processing. Prentice Hall, 1995.

[40] W. Thompson, P. Lechleider, and E. Stuck. Detecting moving objects using the rigidity constraint. PAMI, 15, 1993.

[41] P. Torr. Geometric motion segmentation and model selection. Philosophical Trans. Royal Soc. A, pages 1321-1340, 1998.

[42] S. Ullman. The Interpretation of Visual Motion. MIT Press, 1979.

[43] A. Verri and T. Poggio. Motion field and optical flow: qualitative properties. IEEE Trans. Pattern Analysis and Machine Intelligence, 11(5):490-498, 1989.

[44] Z. Zhang. 3D Reconstruction under Varying Constraints on Camera Geometry for Robotic Navigation Scenarios. PhD thesis, CMPSCI 96-08, University of Massachusetts/Amherst, 1996.

[45] Z. Zhang, R. Deriche, O. Faugeras, and Q.-T. Luong. A robust technique for matching two uncalibrated images through the recovery of the unknown epipolar geometry. Artificial Intelligence, 78:87-119, 1995.

[46] Z. Zhang, R. Weiss, and A. Hanson. Qualitative obstacle detection. In Proc. IEEE International Conference on CVPR. IEEE Computer Society Press, 1994.

[47] Z. Zhang, R. Weiss, and A. Hanson. Obstacle detection based on qualitative and quantitative $3 \mathrm{D}$ reconstruction. PAMI, 19(1), 1997.

[48] Q. Zheng and R. Chellappa. Automatic feature point extraction and tracking in image sequences for unknown camera motion. In Proc. ICCV. IEEE, 1993. 

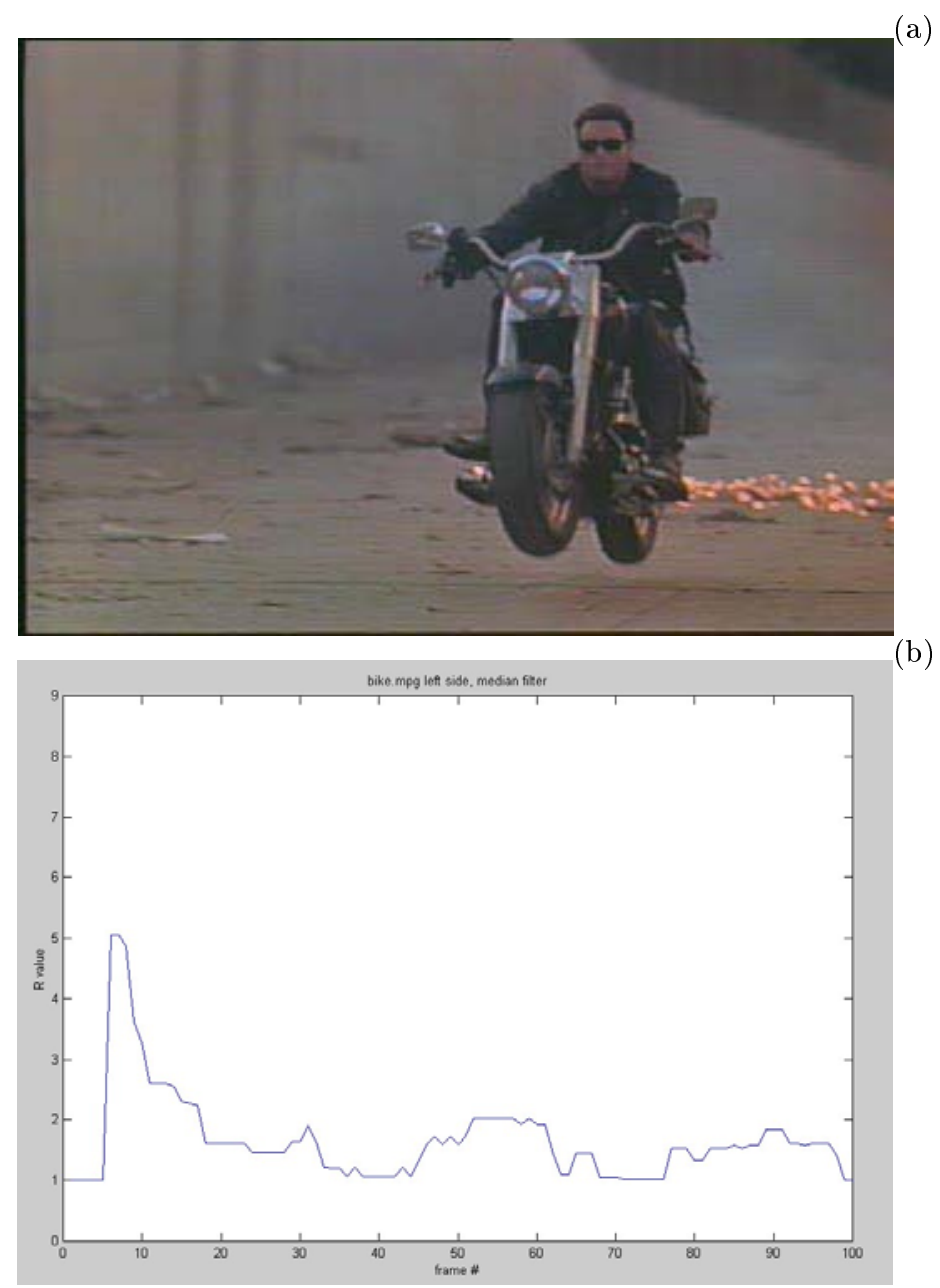

(b)

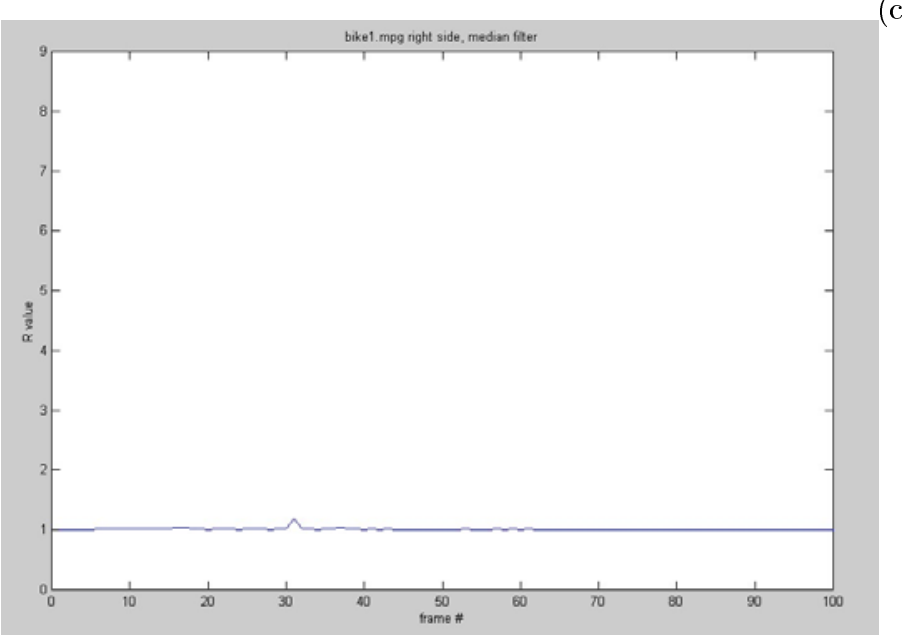

Figure 6: (a) A frame of a movie (100 frames) (b) The median filtered $R$ for the left halves of the video (with no independent motion) (c) The median filtered $R$ for the right halves of the video (with independent motion). 